202 Stenhouse u. Groves, Beiträge zur Geschichte

Beiträge zur Geschichte der Naphtalinreihe; von John Stenhouse und Charles E. Groves.

\title{
B-Naphtochinon.
}

In einer früheren Abhandlung *) erwähnten wir, dafs Nitroso- $\beta$-naphtol nach Umwandlung in die entsprechende Amidoverbindung bei der Oxydation einen krystallinischen Körper von chinonartigem Charakter liefert, den wir $\beta$-Naphtochinon nannten, um ihn von dem von einem von uns früher heschriebenen $\alpha$-Naphtochinon zu unterscheiden. Weitere Versuche haben uns gezeigt, dafs es für die Darstellung dieses Chinons vortheilhaft ist, eine beträchtlich grö ssere Menge Kaliumdichromat anzuwenden, als wir früher benutzten. Empfehlenswerthe Verhalltnisse sind : 6 Th. einer gesāttigten wässerigen Lösung von schwefliger Säure, 6 Th. verdünnte Schwefelsăure (1 Vol. Säure auf 2 Vol. Wasser) und $3 \mathrm{Th}$. Kaliumdichromat auf je $2 \mathrm{Th}$. des reinen Nitroso$\beta$-naphtols; die Reduction und darauffolgende Oxydation werden in der früher beschriebenen Weise ausgeführt (a. a. 0.S. 153). Auf diese Weise liefert das Nitrosonaphtol 62 bis $65 \mathrm{pC}$. seines Gewichtes reines Chinon, während die theoretische Menge $91,9 \mathrm{pC}$. beträgt. Wir konnten den Grund dieses Deficits nicht auffinden : der Schwefel, der sich wăhrend der Reduction des Nitroso- in das Amidoproduct niederschlägt, enthält nur sehr wenig organische Materie; man mufs daher vermuthen, dafs der Verlust hauptsächlich durch theilweise Auflösung des Amido- $\beta$-naphtols in dem verdünnten Schwefelammonium verursacht wird; aber diese Lösung mit Schwefelsāure angesäuert und durch Erwärmen von Schwefelwasser-

*) Diese Annalen $189,145$. 
stoff befreit liefert mit Kaliumdichromat kein Chinon mehr, wie es doch der Fall sein müfste, wenn Amidonaphtol in einiger Menge vorhanden wäre.

\section{Nitro- $\beta$-naphtochinon.}

Es wurde beobachtet, dafs $\beta$-Naphtochinon sich leicht in heifser verdünnter Salpetersäure löst und diese Lösung bei der Abkühlung eine krystallinische Substanz von herrlich scharlachrother Farbe absetzte. Seit unserer !etzten Mittheilung haben wir diese Substatiz rein dargestellt. Reines $\beta$-Chinon (10 Th.) wurde mit Salpetersäure von 1,2 spec. Gewicht (60 Volumtheile) durch Schütteln innig gemischt und in kochendes Wasser eingestellt. Nach einigen Minuten haite sich das Chinon gelōst und die neue Verbindung schied sich in rothen Krystallen aus. Dabei entwickelten sich nur ge-

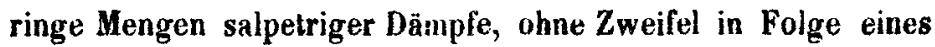
secundären Processes. Nach der vollständigen Umwandlung in das Nitroderivat, welche nur wenige Minuten in Anspruch nahm, wurde die Flasche vom Wasserbad genommen und in kaltes Wasser getaucht, um die oxydirende Wirkung der beifsen Salpetersäure auf das Nitrochinon zu verhindern. Nach metrstündigen Stehen wurde das Product abfiltrirt und zuerst mit verdünnter Salpetersăure (spec. Gewicht 1,2), dann mit Wasser gewaschen. Das Rohproduct betrug 70 bis 75 pC. des in Arbeit genommenen Naphtochinons. Zur Reinigung wurde es im 15 fachen seines Gewichtes kochenden Benzols gel6st und 18 bis 24 Stunden stehen gelassen, wobei es sich langsan in harten rothen, an den Gefälswänden anhängenden Körnern abschied; zuletzt wurden diese noch zwei- bis dreimal aus ihrem doppelten Gewicht kochenden Eisessigs umkrystallisirt, aus welchem das Nitronaphtochinon bein Erkalten in herrlichen rothen, im Aeufsern ganz an Cbromsäureanhydrid 
erinnernden Krystallen anschofs. Bei der Anaiyse wurden folgende Zahlen erbalten :

1. 0,801 Grm. gaben $0,654 \mathrm{CO}_{4}$ und $0,075 \mathrm{H}_{8} \mathrm{O}$.

II. $0,197 n, 0,429 n n 0,046$,

III. 0,427 Grm. gaben 25,4 CC. $N$ bei $18,5^{\mathrm{C}}$ und $764,8 \mathrm{MM}$. Druck (corrigirt für $0^{\circ}$ ), entsprechend $23,448 \mathrm{CC}$, trockenem Stickstoff bei $0^{\circ}$ oder $0,029408 \mathrm{Grm}$.

IV. 0,364 Grm. gaben 21,5 CC. $N$ bei $17,5^{\circ}$ und 745,9 MM. Druok (corr. für $0^{\circ}$ ), entsprechend $19,830 \mathrm{CC}$. trockenem Stickstoff bei $0^{\circ}$ oder 0,024877 Grm.

Diese Zahlen entsprechen der Formel des Mononitro- $\beta$-naphtochinons $\mathrm{C}_{10} \mathrm{H}_{5}\left(\mathrm{NO}_{2}\right) \mathrm{O}_{2}$.

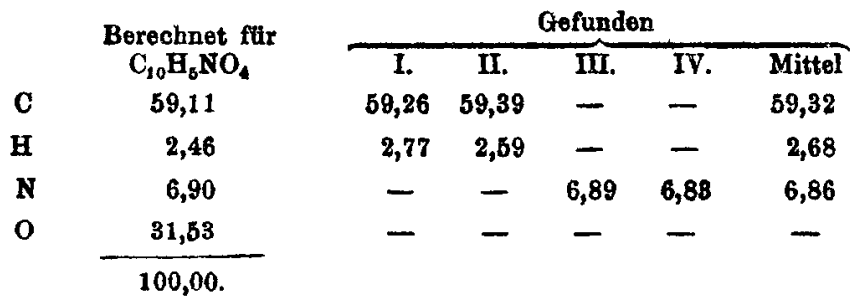

Es wurde bereits gezeigt (a. a. 0.), dafs das Nitro- $\beta$ naphtochinon Phtalsäure bei der Oxydation liefert, so dafs sowohl die $\mathrm{NO}_{\mathrm{g} g r u p p e}$ als die zwei Sauerstoff des Chinons sich in ein und demselben Benzolring befinden nưssen (G räb e's graphische Naphtalinformel zu Grunde gelegb), aber über die relative Stellung dieser Radicale wissen wir nichts. Es ist jedoch nicht unwahrscheinlich, dafs wenn das $\beta$. Chinon sich wirklich als ein Metaderivat herausstellen sollte, das Nitrochinon als $0: 0: \mathrm{NO}_{2}=1: 3: 4$ befunden werden wird; aber gegenwärtig ist so wenig über die Ordnung bekannt, in welcher die Wasserstoffatome im Naphtalin ersetzt werden, dafs diefs noch als rein hypothetisch betrachtet werden mufs.

Nitro- $\beta$-naphtochinon ist unlōslich in Petroleumäther, fast unlöslich in Schwefelkohlenstoff, wenig in Aether, mehr in Benzol und kochendem Alkohol und sehr leicht in heifsem Eisessig löslich. B eim Kochen mit Alkohol seheint es sich zu 
zersetzen, da es beim Abkühlen nicht wieder auskrystallisirt und beim Verdunsten der Lösung gelbliche Oeltropfen abgeschieden werden.

Es löst sich auch ziemlich leicht in kochender Essigsäure von $30 \mathrm{pC}$. (spec. Gewicht 1,04) und in geringerer Menge in Wasser. Diese orangerothen Lösungen worden opalisirend beim Erkalten, aber liefern keine Krystalle. In concentrirter Schwefelsäure lōst sich das Nitrochinon in der Kälte unter Zersetzung; die dunkle Lösung giebt mit Wasser verdünnt einen braunen Niederschlag. Das Nitrochinon schmilzt bei $158^{\circ}$ und verpufft schwach, wenn es auf Platinblech stark erhitzt wird. Durch Jodwasserstoff und Phosphor wird es reducirt; wahrscheinlich entstehen dabei das entsprechende Nitrotydrochinon und Amidohydrochinon, welche in grofsen hochrothen Tafeln und rothbraunen Nadeln krystallisiren und bis jetzt nicht näher untersucht wurden. Wässerıge schweflige Saure reducirt das Nitrochinon ebenfalls.

\section{Dinaphtyldichinhydron.}

Bei der Darstellung des $\beta$-Naphtochinons wurde bemerkt, dafs das frisch niedergeschlagene Chinon beim längeren Stehen in der sauern Lösung seine schöne Orangefarbe verlor und dunkler wurde; dieses schrieben wir der Oxydation durch das im Ueberschufs angewandte Kaliumdichromat $\mathrm{zu}$, fanden. aber jetzt die Ursache in etwas anderem. Wenn das Chinon mit Mineralsāuren erhitzt wird, oder auch nur bei gewöhnlicher Temperatur hinlãngliche Zeit damit in Berübrung bleibt, wird es in einen dem Chinhydron ähnlichen, rōthlich-schwarzen Körper verwandelt und diesem Procefs ist die eben erwähnte Furbenänderung zuzuschreiben, nicht der Oxydation.

Diese Umwandlung des $\beta$-Naphtochinons wird zweckmäfsig mittelst verdünnter Schwefelsāure (1 Vol. Săure auf 
206 Stenhouse u. Groves, Beiträge zur Geschichte

2 Vol. Wasser) bewirkt. Reines Chinon (1 Th.) mit verdünnter Schwefelsäure (10 Volumtheile) wohl durchfeuchtet, war nach 24 Stunden vollständig in jene schwarze Substanz verwandelt. Schneller wurde das Ziel durch Erwärmen auf $\mathbf{5 5}^{0}$ erreicht, wobei das Chinon sich rasch dunkel färbte und nach 10 Minuten der Procefs vollendet war.

Wenn man die Temperatur viel über $60^{\circ}$ steigen liefs, wurden klebrige weiche Massen gebildet, die viel unverändertes $\beta$-Chinon einschlossen, so dafs es etwas schwierig wurde, das Chinon vollständig in das neue Product zu verwandeln. Nach dem Erkalten wurde ungefähr das doppelte Volurn Wasser zugefügt und das Product gut mit kaltem Wasser gewaschen, um die anhängende Schwefelsăure zu entfernen. Das so bereitete Dichinhydron stellt trocken ein blauschwarzes Pulver dar, unlöslich in Wasser, Schwefelkohlenstoff und Petroleumäther, fast unlöslich in Benzol, aber löslich in Eisessig. Die rothbraune Lösung wird durch schweflige Säure sofort entfärbt. Es löst sich auch in concentrirter Schwefelsäure mit tiefgrüner Farbe und wird auf Zusatz von Wasser gefällt.

Durch reducirende Agentien wie Jodwasserstoff und Phosphor oder wässerige schweflige Säure wird das Dichinhydron in farblose Nadeln verwandelt, während es mit oxydirenden Mitteln, wie Salpetersäure oder Kaliumdichromat und Schwefelsäure orangefarbene Prismen liefert, so dafs also diese drei Substanzen in derselben Beziehung zu einander stehen, wie grünes Chinhydron, Hydrochinon und Chinon in der Benzolreihe.

\section{Dinaphityldichinon.}

Das eben beschriebene Chinhydron wird durch Kochen mit Salpetersäure oder mit einer Lösung von Kaliumdichromat und Schwefelsäure leicht in Dinaphtyldichinon verwandelt; 
zur Darstellung des letzteren wird eine Lösung von Dinaphtyldichinhydron in kochender 30 procentiger Essigsäure in Salpetersäure (spec. Gewicht 1,45) eingegossen. Das Dichinon fält in kleinen glänzenden Prismen von prachtvoller Orangefarbe nieder. Giebt man umgekehrt die Salpetersaure in die Essigsäurelösung, so hat der Niederschlag eine schmutzige Färbung, wahrscheinlich wegen der Beimengung von etwas Dichinhydron.

Aus den Analysen des bei $100^{\circ}$ getrockneten Products ergiebt sich die empirische Formel $\mathrm{C}_{10} \mathrm{H}_{5} \mathrm{O}_{3}$, welche in $\mathrm{Hin}$ sicht auf die Entstehung des Körpers zu verdoppeln, also $\mathrm{C}_{20} \mathrm{H}_{10} \mathrm{O}_{4}$ zu setzen ist.

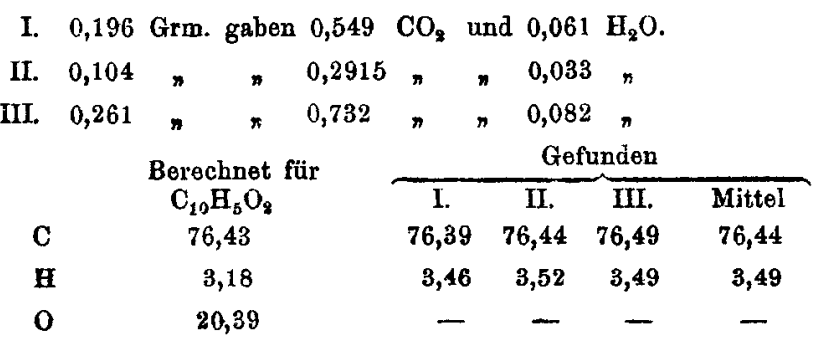

Das Dinaphtyldichinon ist unlöslich in Wasser und nur sehr wenig löslich in den gewöhnlichen Mitteln, sogar kochender Bisessig löst es nur in sehr geringer Menge; es ist ein sehr beständiger Körper, es löst sich in kochender Salpetersäure und krystallisirt beim Erkalten daraus unverändert; doch bei längerem Kochen scheint es zersetzt zu werden. Es löst sich leicht in warmer concentrirter Schwefelsäure und wird daraus beim Verdūnnen wieder gefällt. Reductionsmittel verwandeln es in das correspondirende Hydrochinon.

\section{Dinaphtyldichinol oder Dinaphtyldihydrochinon.}

Diese Verbindung wird durch Reduction des Dichinons oder Dichinhydrons gebildet entweder beim Kochen nit Phosphor und Jodwasserstoff in Essigsäure von $30 \mathrm{pC}$, oder durch 
208 Stenhouse u. Groves, Beiträge zur Geschichte

Behandlung mit wâsseriger schwefliger Säure; das Chinon scheint jedoch leichter reducirt zu werden, als das Chinhydron. Das Dichinon wird bei längerem Stehen mit wässeriger schweffiger Säure in farblose Krystalle des Dichinols verwandelt; ein Chinhydron scheint dabei als Uebergangsstadium nicht gebildet zu werden, da die schwarzen Krystalle dieser Verbindung in der Mischung nie erkannt werden konnten. Sehr leicht erhält man das Dichinol aus $\beta$-Naphtochinon, indem man letzteres durch Behandlung mit verdünnter Schwefelsäure in der vorher beschriebenen Weise in Dichinhydron verwandelt, nach dem Erkalten etwa das doppelte Volum gesättigte wässerige schweflige Săure zusetzt und 24 Stunden stehen lăfst; das schwarze Dichinhydron ist alsdann vollständig in schneeweifse Nudeln von Dinaphtyldihydrochinon verwandelt, vorausgesetzt dafs das ursprüngliche $\beta$-Naphtochinon ganz rein war. Man hat dasselbe nur durch Waschen mit Wasser von Schwefelsäure zu befreien und aus mit etwas schwefliger Săure versetzter verdūnnter Essigsäure umzukrystallisiren, um es vơllig rein zu bekommen. Bei Anwendung unreinen $\beta$-Chinons ist das Product gefärbt und schwer zu reinigen. Wiederholtes Oxydiren zu Dichinon und wieder Reduciren zu Dihydrochinon reicht jedoch gewöhnlich hin, es völlig zu entfärben.

Dinaphtyldichinol $\mathrm{C}_{20} \mathrm{H}_{14} \mathrm{O}_{4}$ oder $\mathrm{C}_{20} \mathrm{H}_{20}(\mathrm{OH})_{4}$ bildet farblose Nadeln, welche beim Trocknen durch theilweise Oxydation dunkel werden. Es schmilzt bei $\mathbf{1 7 6}$ bis $\mathbf{1 7 8}^{\circ}$, ist fast unlöslich in Wasser, mälsig löslich in 30 procentiger Essigsăure, leicht löslich in Eisessig, dagegen nur sehr wenig in Benzol, Schwefelkohlenstoff und Aether. Die analytischen Resultate waren wie folgt :

0,244 Grm. gaben 0,676 $\mathrm{CO}_{2}$ und $0,0905 \mathrm{H}_{2} \mathrm{O}$.

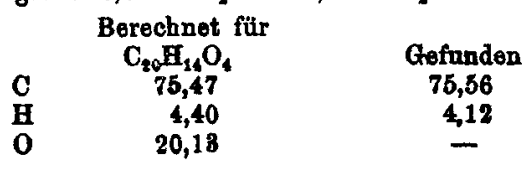


Die Analyse ergiebt etwas zu wenig Wasserstoff für die Formel $\mathrm{C}_{80} \mathrm{H}_{14} \mathrm{O}_{4}$, was bei der leichten Oxydirbarkeit des Körpers kaum anders zu erwarten war.

\section{Bildung des Dinaphtyldichinhydrons.}

Die Umwandlung des $\beta$-Naphtochinons in das schwarze Dichinhydron durch Einwirkung von Säuren scheint unter Condensation von zwei Chinonmoleculen stattzufinden, indem zwei Atome Wasserstoff aus den Benzolkernen eliminirt werden und sich mit zwei von den vorhandenen vier Sauerstoffatomen vereinigen :

$$
\mathrm{C}_{10} \mathrm{H}_{6} \mathrm{O}_{2}+\mathrm{C}_{10} \mathrm{H}_{8} \mathrm{O}_{3}=\mathrm{C}_{90} \mathrm{H}_{40} \mathrm{O}_{8}(\mathrm{OH})_{2}
$$

oder wenn man das Chinhydron durch Vereinigung eines Moleculs Chinon mit einem Molecul Hydrochinon entstanden denkt :

$$
2 \mathrm{C}_{10} \mathrm{H}_{8} \mathrm{O}_{2}+2 \mathrm{C}_{40} \mathrm{H}_{6} \mathrm{O}_{2}=\mathrm{C}_{90} \mathrm{H}_{40} \mathrm{O}_{4} \cdot \mathrm{C}_{20} \mathrm{H}_{10} \mathrm{O}_{2}(\mathrm{OH})_{2} \text {. }
$$

Letztere Ansicht wird sehr gestützt durch die Thatsache, dafs $\beta$-Naphtochinon sein eigenes Gewicht an Dichinhydron liefert. Bei zwei Versuchen wurde aus je $0,500 \mathrm{Grm}$. reinem $\beta$-Naphtochinon durch Behandlung mit verdünnter Schwefelsäure nach dem Waschen und Trocknen 0,498 und 0,495 Grm. Dichinhydron erhalten.

Bei dem hohen Atongewicht dieser Substanzen und der leichten Oxydirbarkeit des Chinols läfst sich durch die Elementaranalyse allein nicht entscheiden, ob unsere Auffassung von der gegenseitigen Beziehung dieser Körper die richtige ist. Es war uns daher sehr erwünscht eine Reaction aufzufinden, durch welche die Anzahl der Wasserstoffatome aufserbalb des Kerns bestimmt werden kann. Bromwasser oxydirt mit grofser Leichtigkeit das Dichinhydron, während es das Dichinon nicht angreift.

Eine gewogene Menge des reinen $\beta$-Naphtochinons wurde demgemäfs in das schwarze Dichinhydron verwandelt und mit 
überschüssigem Bromwasser von bekannter Stärke einige Minuten geschüttelt, wobei es in das Dichinon verwandelt wurde. Das überschüssige Brom wurde dann mit Jodkalium und Hyposulfit in der gewöhnlichen Weise titrirt. Bei zwei Versuchen wurde so gefunden, dafs $0,200 \mathrm{Grm}$. des reinen $\beta$-Naphtochinons nach der Umwandlung in das Dichinhydron 0,100 und 0,098 Brom bedurften, um das neue Chinon zu bilden, während die Gleichung :

$$
\mathrm{C}_{20} \mathrm{H}_{10} \mathrm{O}_{2}(\mathrm{OH})_{2}+\mathrm{Br}_{3}=\mathrm{C}_{20} \mathrm{H}_{16} \mathrm{O}_{4}+2 \mathrm{HBr}
$$

0,101 Grm. Brom verlangt. Es kann daher kaum mehr ein Zweifel bestehen, dafs die schwarze Substanz ein dem grünen Chinhydron der Benzolreihe entsprechendes Dichinhydron ist.

In gleicher Weise wurde das Dichinol oder Dihydrochinon mit Bromwasser oxydirt; die Resultate stimmen hier nicht so genau überein, da das Dichinol zu rasch durch den Sauerstoff der Luft Oxydation erleidet.

Eine gewogene Menge Dinaphtyldichinon $\mathrm{C}_{20} \mathrm{H}_{10} \mathrm{O}_{4}$ wurde mit schwefliger Säure reducirt; nach sorgfältigem Auswaschen auf dem Vacuumfilter, wobei sich das Product schon ein wenig färbte, wurde es in heirsem Eisessig gelöst und die etwas dunkel gefärbte Lösung in Bromwasser gegossen; den Bromüberschufs tritrirten wir wie oben. Drei Versuche ergaben $98,88,5$ und 85 pC. Brom auf $100^{\circ}$ Dichinon, während die Theorie 101,9 pC. verlangt. Das bei diesen Oxydationsversuchen gebildete Dichinon wurde auf einen Gehalt an Bromsubstitutionsproducten geprüft, aber frei von Brom gefunden.

Die Verbindung $\mathrm{C}_{20} \mathrm{H}_{10}(\mathrm{OH})_{4}$ kann als ein vieratomiger (tetrahydrischer) Alkohol der Hydrochinon- oder Chinolklasse, hervorgegangen durch Substituirung von vier Wasserstoffatomen in einem Dinaphtyl durch vier Hydroxyle betrachtet werden und ist ein Glied einer neuen Körperklasse, der Dichinole. Wir nannten inn deshalb Dinaphtyldichinol und das entsprechende Chinon Dinaphtyldichinon. 
Wir haben ferner gefunden, dafs Benzochinon und $a-N a p h t o c h i n o n$ ebenfalls Dichinene und Dichinole durch Condensation liefern und bezweifeln nicht, dafs aus anderen Chinonen entsprechende Verbindungen erhalten werden können. Wir sind gegenwärtig beschäftigt, diese interessante Gruppe von Körpern zu studiren.

\section{Ueber die Einwirkung von saurem chrom-} saurem Kali und Schwefelsäure auf Cholsäure;

\section{von Dr. H. Tappeiner,}

Privatdocent und Assistent am pathologischen Institute in Mtinchen.

(Aus dem Laboratorium des pathologisohen Instituts in München.)

(Der kaiserl. Academie d. Wissensch. zu Wien vorgelegt in der Bitzung am 4. April 1878.)

Die durch diese Reagentien aus Cholsäure (synonym Cholalsäure, Str ecke r) erhaltenen, rein dargestellten Körper, von einigen flüchtigen und nicht flüchtigen, aber nicht genügend isolirten Säuren der Fettsäurereihe abgesehen, sind : Cholesterinsäure $\mathrm{C}_{78} \mathrm{H}_{16} \mathrm{O}_{7}$, Stearinsäure, Laurinsäure und Cholansãure $\mathrm{C}_{20} \mathrm{H}_{28} \mathrm{O}_{6}$ *). Die Mischung von Schwefelsäure, saurem chromsaurem Kali und Wasser kam entweder in dem von

*) Vorlăufige Mittheilnngen sind in den Berichten der deutschen chemischen Gesellschaft 1873 und in der Zeitschrift für Biologie 1876 erschienen. Cholansäure ist dort mit $A$, Cholesterinsăure mit $B$ bezeichnet. 